\title{
THE RELATIVISTIC AND THE HIDDEN MOMENTUM OF MINKOWSKI AND ABRAHAM IN RELATIVISTIC ENERGY WAVE
}

\author{
Daniel Souza Cardoso \\ Instituto Federal Sul Rio-grandense, Campus CaVG, Pelotas, Brasil \\ danielcardoso@cavg.ifsul.edu.br
}

\begin{abstract}
An analysis of the consistency of the Abraham and Minkowski momenta in the determination of the photon trajectory was carried out considering a new principle of conservation of the photon's mechanical energy, in which the photon conserves translational energy in orbital angular momentum when transiting between two media, introducing the relativistic energy wave (REW). The confrontation between REW and the recent theory of space-time waves (ST) was considered, pondering your differences. Throughout this study it was possible to verify that the Abraham momentum appears a relativistic photon ignition device in the transition between two media, acting as the hidden momentum of the Minkowski's relativistic momentum. The wavy behavior in the matter is relativistic, and the relativistic trajectory appears with delays and advances, with points of synchronization between source-observer. The classical or relativistic trajectories are determined as a function of the angle of incidence and the relative refractive index, by one of two distinct non-additive torques, the classic by Abraham or the relativistic by Minkowski. It was found that the same analysis conducted under the principle of conservation of the mechanical energy of the photon can be treated by an new Doppler, Relativistic Apparent, that can be confused with other Dopplers in the treatment of redshift from distant sources. It was found that the conservation of energy in Orbital Angular Momentum (OAM), in the interaction with matter, explains that the synchronization instants are found in the inversion of the OAM, where the advances and delays of REW occur under negligible variations of the OAM, however, opposites.
\end{abstract}

Keywords: Photon, Relativistic Energy Wave, Abraham Momentum, Minkonwski Momentum, Relativistic Photon Ignition, Apparent Doppler, Orbital Angular Momentum, OAM.

\section{INTRODUCTION}

Countless phenomena that characterize the properties of light are known and well established in the literature, whether in corpuscular or wavy treatment. However, the photon's behavior is the center of a series of discussions that has been going on for decades, such as the 
redshift [34], [15], [12], of the controversial momenta between Abraham and Minkowski [16], [7], [38], and sometimes it was thought that light got tired [21].

The Abraham and Minkowski momenta are protagonists of the photon momentum in dielectrics, which, although distinct, respond correctly to experiments that support one or the other [3], [2], [31], [39]. They disagree on the intensity of the photon momentum in dielectrics, as for Minkowski the linear momentum of the photon increases in the dielectric media in proportion to the index of refraction of the media, n, while Abraham's momentum characterizes a decrease in the linear momentum of the photon, in inverse proportion to the index of refraction.

Recently, Barnett [3] characterized the Minkowski momentum as canonical and the Abraham momentum as the kinetic momentum, because although the Abraham momentum is shown to be more theoretically adjusted to the conservation of global kinetic moment, the Minkowski momentum is adjusted to the quantum theory. The Abraham and Minkowski momenta are consistent with the De Broglie momentum, which in turn characterizes the wavelength of a wave of matter [18].

The hidden momentum appears in the scope of the description of the movement of magnetic dipoles subject to external fields, in refracting means, where the hidden resultant is the relativistic momentum itself [19], which in turn allows the dipole to have a hidden momentum even when at rest. The author concludes that Abraham's momentum is associated with the kinetic part, while the Minkowski's is associated with the canonical part. According to Saldanha [38], the separation of the momentum of the electromagnetic wave in electric and magnetic media, is consistent in electromagnetic and material parts, where the hidden momentum characterizes the momentum of Abraham.

Curtis and Grier [17], showed that a conventional beam can be modulated through mode converters, obtaining helical modes, originating the optical vortices. The authors present a model in which they describe that the movement of a photon around the vortex has direct contributions to the angular momentum of the beam, reporting that there is an increase in power with a reduction in velocity, in the perception that the more it is pushed, the slower they become.

The conservation of angular momentum in the photon-matter interaction was discussed in recent study, where Chang et al. [47] show that an electron subjected to microwave radiation endowed with OAM will experience the balance of the conservations of linear and angular momenta. The authors found that the photon-matter interaction, considering photons initially endowed with OAM, can generate electron vortices, with different quantum numbers.

Ji et al. [27] designed a photodetector to analyze the OAM of light where they verified that in the photon-matter interaction, the electron can simultaneously acquire energy and OAM from the incident light, inducing a spatial imbalance of the carriers involved. The authors gave the name of 
orbital photogalvanic effect to the induced current, due to its distinction, whereas it originates from the OAM of light.

Schmiegelow et al. [42], considering that in the photon-matter interaction an OAM carrying a photon can transfer OAM to a free electron, conducted a study analyzing the transference of OAM to a bound electron, demonstrating the transference of OAM and spin angular momentum, SAM, from the field to the ion.

According to Forbes et al. [22], the total angular momentum is composed of the OAM momenta [1] and SAM, where the SAM is present, regardless of the presence of a material media, conserving the helicity which, in its turn, is not transferable to the matter. The OAM by photon along the propagation axis characterizes the movement of a particle around the propagation axis is transferable to matter. The OAM features a cross-beam structure, where the experience [23] demonstrates that the transverse structure causes a delay in the average speed of photons in a beam, when compared to a beam without structure, even in air.

In a theoretical analysis of the corpuscular behavior of light, Cardoso [13] introduces a principle for the conservation of the photon's mechanical energy, where the photon transiting between two media conserves translational energy in OAM, shifting its wavelength. Later [10], this wave displacement is verified in the prediction of interference fringes comparing to experimental data from a Michelson interferometer, verifying that the model according to the principle of conservation of the photon mechanical energy presents estimative with smaller percentage of uncertainties $(<0.5 \%)$ and smaller percentage of deviations $(<2.0 \%)$ when compared to the literature model estimative in the data representation.

Recently, Cardoso [12] demonstrates the nature of the redshift according to the principle of conservation of the mechanical energy of the photon, presenting the gravitational redshifts ( $\mathrm{z}_{\mathrm{CEF}-\mathrm{G}}$ ) and refractive $\left(\mathrm{z}_{\mathrm{CEF}-\mathrm{G}}\right)$ which individually are able to describe the gravitational and cosmological redshifts keeping constant the Hubble constant. However, the author considered the composition of all the effects capable of promoting a displacement of the light's wavelength, possibles in the trajectory, presenting a balance through the redshift $Z_{\mathrm{CEF}}$, considering the Doppler effect, demonstrating that in the absence of the principle of conservation of mechanical energy, the Hubble constant must vary by small variations in the relative velocity between source-observer.

In this analysis verified the consistency between Abraham and Minkowski momenta in the determination of the photon trajectory, and its implications regarding the incidence angle and relative refractive index, considering the conservation of the photon's mechanical energy in rotational energy. 
A comparative analysis was conducted between the relativistic behavior of the photon and the recent space-time (ST) waves, in the perspective of delays between the source and observer perceptions. It was verified the existence of refraction possible anomalies in the perspective of a single photon, in an analogue weighing the differences with those recently associated with group speeds [6], [33], [46]. The relationship between the variation of the OAM and the delays between the source-observer perceptions was verified, in the perspective of the conservation of the mechanical energy of the photon.

\section{METHODOLOGY}

The analysis considers the transmission of a single photon in the transition between pairs of media previously known in the literature, with regard to refractive properties. The refraction data established in the literature and the Laws of Refraction are compared to verify the variability of the data estimated by the proposed models.

In the corpuscular treatment of the photon-matter interaction, the conservations of energy, linear momentum, and angular momentum, were considered. In the characterization as an energy package, the quantization of energy and the uncertainty principle were considered. The analysis of the state of movement considered a relativistic description consistent with the behavior of the data.

\section{DEVELOPMENT AND DISCUSSION}

We will assume that the photon far from a vacuum behaves like a material wave of energy, where its state of movement is characterized by the De Broglie momentum [18]:

$$
p=\frac{h}{\lambda} \text {, }
$$

although clearly it's about the quantized quantity, we can satisfy that there is a classic part that can be described by the Snell-Descates Law [36] $\left(\lambda \mathrm{n}_{21}=\lambda_{0}\right)$ :

$$
\Delta \lambda=\lambda\left(1-n_{21}\right) \text {, }
$$

so we can write:

$$
\Delta p=\frac{h}{\Delta \lambda}\left(1-n_{21}\right),
$$

where the wavelength shift in the transition between two media:

$$
\Delta \lambda=\frac{h}{\Delta p}\left(1-n_{21}\right) .
$$

In the interaction with matter, the unabsorbed or scattered photons interact with electrons at short distances and without contact, characterizing an elastic collision. In the model of Compton, the electron was at rest before the interaction, but in agreement with Cardoso [13] the electron has linear and angular momenta before the interaction, where the conservation of linear momentum: 


$$
\Delta \vec{p}_{\text {electron }}=-\Delta \vec{p}_{\text {photon }} .
$$

In accordance with the literature [20], when an electron is found in a region delimited by $\mathrm{r}$, the moment uncertainty:

$$
\Delta p=\frac{h}{2 \pi r},
$$

where each component of linear momentum carries i can a uncertainty, so that we can approximate:

$$
\Delta p=p,
$$

satisfying that we can represent increases or decreases in the mechanical energy of the electron, in terms of the variation of the linear momentum of the photon, considering relations (5) and (7):

$$
\left|\Delta E_{\text {electron }}\right|=\frac{1}{2 m_{e}}\left(\Delta \vec{p}_{\text {photon }}\right)^{2},
$$

so that the displacement of the photon's wavelength in part characterizes the De Broglie wavelength shift [18] in the description of matter waves, such that replacing (8) in (4):

$$
\Delta \lambda=\frac{h}{\sqrt{2 m_{e}\left|\Delta E_{\text {electron }}\right|}}\left(1-n_{21}\right) .
$$

The variation of the photon's linear momentum has an associated energy that should make up the balance of the conservation of the photon's mechanical energy:

$$
m c^{2}=m v^{2}+\Delta E_{\text {photon }},
$$

where the increase in energy:

$$
\Delta E_{\text {photon }}=m c^{2}\left(1-\frac{v^{2}}{c^{2}}\right),
$$

considering again the Snell-Descates Law and in terms of the quantized energy of the photon:

$$
\Delta E_{\text {photon }}=h v\left(1-\frac{n_{1}^{2}}{n_{2}^{2}}\right) \text {. }
$$

In the interaction with matter, the unabsorbed or scattered photons interact with electrons at short distances and without contact, characterizing an elastic collision where the intensities agree:

$$
\left|\Delta E_{\text {electron }}\right|=\left|\Delta E_{\text {photon }}\right|,
$$

such that the displacement of the wavelength characterizes the new principle of conservation of mechanical energy for the photon acoording Cardoso [13], [10], [12]:

$$
\Delta \lambda=\frac{h}{\sqrt{2 m_{e} h v\left(1-n_{12}^{2}\right)}}\left(1-n_{21}\right) .
$$




\subsection{PHOTON RELATIVISTIC IGNITION}

The expression (14) demonstrates that the wavelength shift is relativistic ${ }^{1}$ :

$$
\Delta \lambda=\frac{1}{\sqrt{1-\frac{v^{2}}{c^{2}}}} \frac{h}{\sqrt{2 m_{e} h v}}\left(1-n_{21}\right),
$$

according to Saldanha [38] there is a material part, where the De Broglie wavelength can now represent this amount:

$$
\lambda_{\text {Broglie }}=\frac{h}{\sqrt{2 m_{e} h v}},
$$

simplifying the expression (15):

$$
\Delta \lambda=\gamma \lambda_{\text {Broglie }}\left(1-n_{21}\right),
$$

where it is noted that the De Broglie wavelength dilation $(\gamma \lambda)$ is a consequence of the variation of the photon's energy, associated with the angular momentum acquired by the photon in the interaction:

$$
\Delta \vec{L}_{\text {electron }}=-\vec{L}_{\text {photon }},
$$

satisfying that the photon before the interaction had only linear momentum.

Considering the angular momenta of Abraham and Minkowisk for the photon [39], we can write:

$$
L_{\text {Minkowski }}-L_{\text {Abraham }}=I \hbar\left(1-n_{12}^{2}\right),
$$

where "l" is the Laguerre-Gauss mode.

The energy given by equation (12) can be associated with angular momentum due to the torque associated with Abraham and Minkowski momenta:

$$
\frac{1}{2} \omega L=h v\left(1-n_{12}^{2}\right)
$$

where the eq. (20), considering eq. (19) reveals a structure ${ }^{2}$ of the photon movement where analogue $\mathrm{l}=2$ is consistent with other studies [26], although we will verify in section 3.4 that a more peculiar modulation appears in the photon-matter interaction. Naturally satisfying that the photon conserves its energy in a form of movement, for if the increment in energy is transferred for media, it will soon be dissipated. The refraction process, for example, is a process in which the photon transits between two media with constant frequency and naturally conserves energy in forms of movement.

1 The equation (15) will only have a relativistic character if the speed of the photon in the refringent media (in the second term of the radical) is the relative speed between two reference frames. Here we follow the analysis previously treating it as relativistic, although this criterion is discussed later in section 3.2.

2 In section 3.4 the modulations are presented as a function of the relative refractive index and the incidence angle. 
The increment in energy conserved in OAM implies in a decrease in linear momentum, which is now characterized by Abraham's $\mathrm{s}^{3}$ momentum $\left(\mathrm{hn}_{12} / \lambda\right)$, acting as a relativistic ignition device that drives the photon into a relativistic movement:

$$
\Delta p=\gamma \frac{h}{\Delta \lambda}\left[1-n_{21}\right],
$$

explaining a greater moment in the refringent environment, center of the Abraham-Minkowski conflict, resultant from a dilation of the moment. We can treat the Mikonwski momentum as a relativistic momentum in the material refringent:

$$
p_{\text {Minkowski }}=\gamma \frac{h}{\Delta \lambda} n_{21} \text {. }
$$

Characterized, the relativistic moment in accordance with the principle of energy conservation of photon's mechanics [13], [10], [12], with representation of the Mikonwski momentum, we can verify the relationship directly from the Mikonwski momentum itself [3], it is:

$$
p\left(n_{21}\right)=\frac{h}{\lambda_{0}} n_{21}
$$

considering the relation (7), we can write:

$$
\lambda=\frac{h}{\Delta p} n_{21}
$$

similarly, considering again the Snell-Descates Law:

$$
\lambda_{0}=\frac{h}{\Delta p},
$$

where in the subtraction between eq.(s) (23) and (22) eq. (4), of which following the same analysis of eq. (s) $(8,9,12$ and 13) results in eq. (14).

The eq. (15) shows that the displacement of a relativistic wave for photon results from the decrease in energy associated with the translational movement. Abraham's linear momentum (hn12/ $\lambda$ ) characterizes a hidden momentum, according to Saldanha [38], being now the hidden momentum of Mikowski's relativistic momentum.

The material part of the eq. (15) shows agreement with Goray et al. [24], while saying that light as a particle when incident on the separating surface between two media will present a wavy behavior. Here we demonstrate the composition of a material energy wave characterized by the De Broglie momentum, where the photon in the material media has a wavelength and relativistic moment equivalent to that of an electron with energy hv. Tan [44], in a study of the photon's imaginary rest mass, found a relationship similar to eq. (15), where is discussed that the excitation of matter implies a material wave of a particle similar to the electron neutrino, with energy hv.

3 From the analysis of the deformations characterized from equation (25), Abraham's moment becomes more familiar to the reader. 
In this context, we present that the photon, when interacting with the matter, starts to behave in the form of a relativistic energy wave whose linear trajectory can be found through the relativistic deformation of the linear momentum of the wave:

$$
\left[\frac{\Delta p}{p}\right]=\left[\frac{\Delta \theta}{\theta_{1}}\right]=\frac{\left(1-n_{21}\right)}{\sqrt{1-\frac{v^{2}}{c^{2}}}} \cos \left(90^{\circ}-\theta_{2}\right)=-\frac{\left(1-n_{12}\right)}{\sqrt{1-\frac{v^{2}}{c^{2}}}} \operatorname{sen} \theta_{1},
$$

although a classical trajectory is also allowed, which agrees with the Snell'-Descates Law for small angles, and it happens by a decrease for the Abraham momentum:

$$
\left[\frac{\Delta p}{p}\right]=\left[\frac{\Delta \theta}{\theta_{1}}\right]=-\left(1-n_{12}\right) \text {. }
$$

It can be noticed that eq. (25) has a classic character while (24) is relativistic, and it is noticeable that in the latter, the variation of the linear momentum characterizes its projection in the direction perpendicular to the actual path of the photon Figura 1, while the variation of the linear momentum of Abraham represents the deformation of small angles, properly. Although relativistic effects occur in the transition between the two media, the real trajectory to be followed by the photon can be defined by two non-additive torques, as they are in different scenarios, a classic ( $\tau_{\text {Abraham}}$ ) and the relativistic ( $\tau_{\text {Minkowski }}$ ), where we will verify that Minkowski's agrees with the relativistic trajectory that arises with delays, advances, and synchronizations as we will discuss in this analysis. Relativistic delays are found in different scenarios [43], [6], [30].

In the Figura 1 there are two regions defined by the angle of incidence. In the classical region, the incidence up to approximately $41^{\circ}$, the trajectory imposed on the photon in refraction is that determined by the Abraham torque, while in the relativistic region the relativistic trajectory comes up with a certain delay as discussed in section 3.2, determined by the Minkowski torque, for angles of incidence greater than $41^{\circ}$. In this way, the angular deformation for an incidence of up to $41^{\circ}$ :

$$
\left[\frac{\Delta \theta}{\theta_{1}}\right]_{0-41^{\circ}}=-\left(1-n_{12}\right)
$$

For angles greater than $41^{\circ}$, the angular deformation is represented by the deformation of the Minkowski linear momentum according to eq.(24), with characteristics similar to frequency independent rotational Doppler as reported by Martin et al. [32], discussed in the section 3.2, as:

$$
\left[\frac{\Delta \theta}{\theta_{1}}\right]_{41-80^{\circ}}=-\frac{\left(1-n_{12}\right)}{\sqrt{1-\frac{v^{2}}{c^{2}}}} \operatorname{sen} \theta_{1}
$$

where the transition from the deformation regime is around $41^{\circ}$ : 


$$
\left[-\left(1-n_{12}\right)\right]_{41^{\circ}} \approx\left[-\frac{\left(1-n_{12}\right)}{\sqrt{1-\frac{v^{2}}{c^{2}}}} \operatorname{sen} \theta_{1}\right.
$$

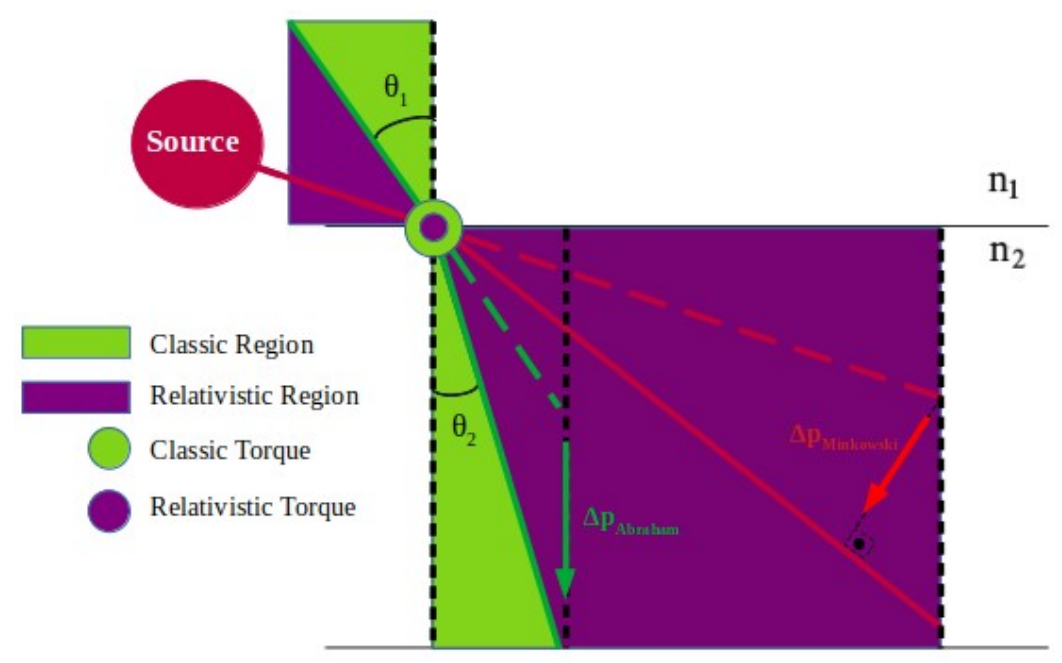

Figure 1- Classical and Relativistic trajectory regions as a function of the angle of incidence. For angles of incidence up to $\sim 41^{\circ}$, the angular deformation is due to the Abraham torque, for larger angles the predominance is Minkowski. The illustrated Minkowski momentum variation represents its projection in the considered direction. (Source: author)

The eq.(s) (26) and (27), treated below as strains, are checked against the refracted angle estimates according to the Snell-Descates Law and the behavior of the data.

Tabela 1 compares the refracted angle estimates between the Snell-Descates Law of sines and the strains given by eq.(s) (26) and (27), noting that the mean deviation of the strain estimates in relation to the data, is eight times smaller than those of Snell-Descates. This comparison was chosen due to the historical fact that the Snell-Descates Law stood out against different models that sought to describe the refraction process, with propositions from Ptolemy and Kepler [9], between others. Notoriously, the deformations are well-fitted, representing the behavior of the data.

Table 1 - Comparison the estimates of the refracted angle in water between the Snell-Descates Law and the linear momentum strains. An air-water transition was considered under the refractive indices 1.0003 and 1.3333, respectively. In dark gray the estimate of eq.(26) and in light gray the estimates of eq. (27). (Source: author).

\begin{tabular}{|c|c|c|c|c|c|}
\hline$\theta_{1}{ }^{*}$ & $\boldsymbol{\theta}_{2}{ }^{*}$ & Estimatives: Snell-Descartes & Estimatives: Deformations & $\begin{array}{c}\text { Deviations Snell- } \\
\text { Descartes }\end{array}$ & $\overline{\text { Deviations: Deformations }}$ \\
\hline 10 & 7,75 & 7,49 & 7,5 & $-0,26$ & $-0,25$ \\
\hline 20 & 15,5 & 14,87 & 15 & $-0,63$ & $-0,5$ \\
\hline 30 & 22,5 & 22,03 & 22,51 & $-0,47$ & 0,01 \\
\hline 40 & 29 & 28,83 & 30,01 & $-0,17$ & 1,01 \\
\hline 50 & 35 & 35,08 & 35,53 & 0,08 & 0,53 \\
\hline 60 & 40,5 & 40,52 & 40,37 & 0,02 & $-0,13$ \\
\hline 70 & 45,5 & 44,83 & 45,15 & $-0,67$ & $-0,35$ \\
\hline \multirow[t]{3}{*}{80} & 50 & 47,63 & 50,24 & $-2,37$ & 0,24 \\
\hline & & & \multicolumn{3}{|c|}{ Deviations means: } \\
\hline & & & & $-0,56$ & 0,07 \\
\hline
\end{tabular}

* Data from $\theta_{1}$ e $\theta_{2}$ can be found in Cardona et al. [9]. 
The Figure 2 present the estimates of the deformations of eq.(s) (26) and (27) and the data the refracted angles, where the trajectories followed by the photon are verified as a function of the angle of incidence. The classical trajectory determined by the torque of Abraham represents the refraction data of water, as shown in Table 1 , for incidences up to approximately $41^{\circ}$, while for larger angles the photon follows the relativistic trajectory determined by the Minkowski torque.

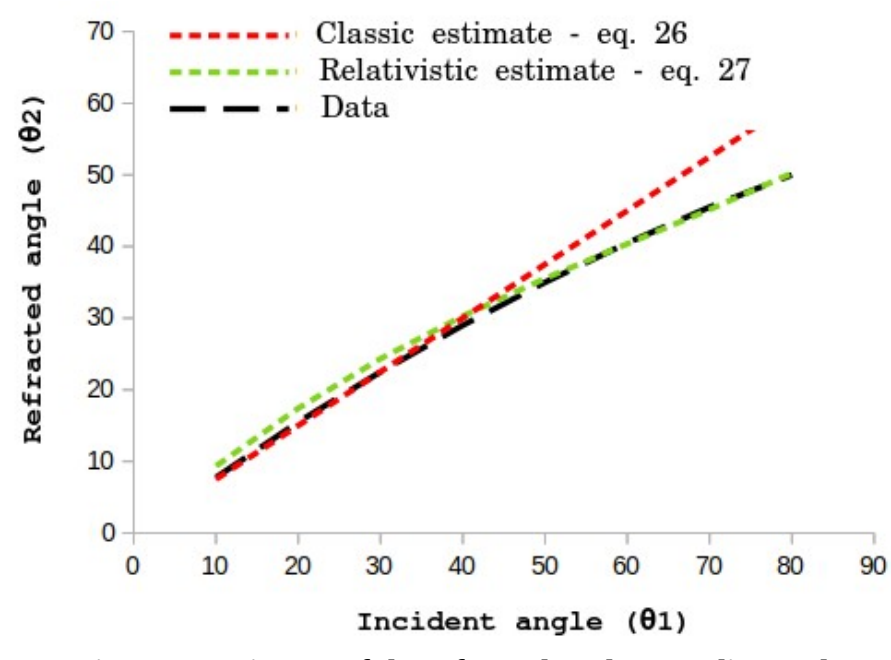

Figure 2- Estimates of the refracted angle according to the deformations and data, acoording table 1. (Source: author)

\subsection{RELATIVISTIC APPARENT DOPPLER}

The deformations presented in eq.(s) (26) and (27) are clear Doppler signatures, which suggests that we have a Doppler effect where source and observer are fixed, differing from the Dopplers translational, transverse, and angular where there is associated to one relative movement. It is a Doppler effect for light, with constant frequency, in which the photon when transiting between two media presents a decrease in its momentum, the effect of which is analogous to the appearance of movement source-observer relative, which we now observe in the example configuration in Figure 3. 


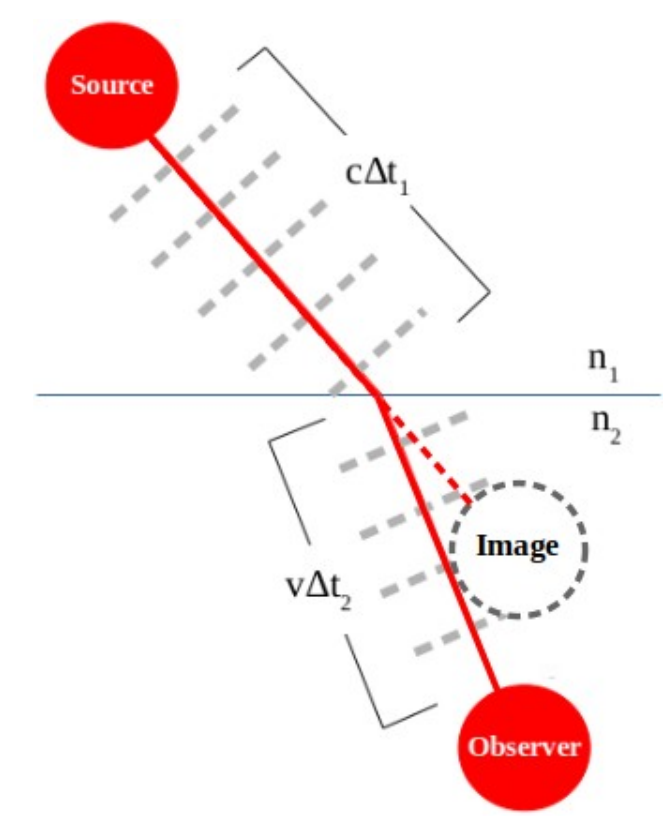

Figure 3- Representation of the wave fronts found in the laboratory, in a refraction process. Gray dashed lines represent wavefronts. (Source: author)

From the observer's perspective, the wavelengths in both media:

$$
\lambda_{1}=\frac{c \Delta t_{1}}{N_{1}} ; \lambda_{2}=\frac{v \Delta t_{2}}{N_{2}},
$$

where $N_{1}$ and $N_{2}$, the numbers of wavefronts associated with the refringent media $n_{1}$ and $n_{2}$, respectively. The wavelength shift recorded by the observer:

$$
\Delta \lambda=(v-c) \frac{\Delta t_{2}}{N_{2}}=\left(1-n_{21}\right) \frac{v \Delta t_{2}}{N_{2}},
$$

where it was considered a process in which the frequency is constant, such as:

$$
\frac{\Delta t_{1}}{N_{1}}=\frac{\Delta t_{2}}{N_{2}}
$$

In a translational Doppler analog, the decrease in the speed of the photon in relation to the observer characterizes an effect of relative movement between source-observer, such that in terms of proper time:

$$
\Delta \lambda=\frac{\left(1-n_{21}\right)}{\sqrt{1-\frac{v^{2}}{c^{2}}}} \frac{v\left(\Delta t_{2}\right)_{\text {proper }}}{N_{2}},
$$

where in accordance with the relations (29), we can:

$$
\Delta \lambda=\frac{\left(1-n_{21}\right)}{\sqrt{1-\frac{v^{2}}{c^{2}}}} \lambda_{\text {Broglie }},
$$

characterizing the displacement presented in eq. (15), the proper wavelength in the material media can be characterized by that of De Broglie. 
An important aspect to characterize the apparent relativistic moviment is that in fact the speed of the photon in $n_{2}$ be the perception of apparent relative velocity between source-observer, although there is no real movement between them. We know that both will have perceptions of apparent positions in relation to the other. In the source referential, the perception of the apparent position of the image the observer allows us to say that the image has moved in a very small amount of time $\left(\Delta \mathrm{t}_{1}+\Delta \mathrm{t}_{2}\right)$ with a very high speed $\mathrm{v}_{\text {image }}=\left(\mathrm{c}^{2}-\mathrm{v}^{2}\right)^{1 / 2}$, projected on the opposite cateto of the triangle featured in Figure 1, where in this case the photon velocity (v) in $n_{2}$ is the apparent relative speed of approximation between source and observer, characterizing an apparent relativistic movement between source and observer. The use of photon speed after transmission as relative speed can be found in other studies [14], [35], [40].

In the Figure 2, we noticed that the relativistic apparent Doppler is predominant for angles greater than $41^{\circ}$. The greater the angle of incidence, the greater the displacement of the image, which implies that the displacement time will be greater since the apparent relative velocity is independent of the angular position, but of the refringence, for both references. In this sense the time is relativistically dilated, with greater effect for large angles, where considering equations (24) and (25), we can write:

$$
[\Delta \theta]_{\text {relativistic }}=\frac{\operatorname{sen} \theta_{1}}{\sqrt{1-\frac{v^{2}}{c^{2}}}}[\Delta \theta]_{\text {classic }},
$$

where, in the perspective of the source's referential, the position of the image moves in an interval:

$$
\left[\frac{\Delta \theta}{|\vec{c}-\vec{v}|}\right]_{\text {source }}=\frac{\operatorname{sen} \theta_{1}}{\sqrt{1-\frac{v^{2}}{c^{2}}}}\left[\frac{\Delta \theta}{|\vec{c}-\vec{v}|}\right]_{\text {observer }} .
$$

Eq. (35) demonstrates that as the angle of incidence increases, time dilation becomes purely relativistic in the perception of the source referential. It can be seen that for angles of incidence up to $41^{\circ}$, the time is contracted in the perception of the source referential, expanding if for larger angles until the next synchronization as will be discussed in Figure 4, which implies a delay in the relativistic trajectory:

$$
[\Delta t]_{\text {delay }}=[\Delta t]_{\text {source }}\left[\sqrt{1-\frac{v^{2}}{c^{2}}}-\operatorname{sen} \theta_{1}\right] \text {. }
$$

Although the last relativistic analyzes took place in the perception of the source referential, the fact that both can describe apparent positions, we could obtain the same results adopting the other observer, in the perspective of the apparent position of the source.

The Doppler characterized in eq. (33) for distant media, it can be confused with other Dopplers if the only criterion is the wavelength shift or redshift. In astronomy, an Apparent Doppler 
can lead to inaccurate distances and velocities of systems or stars. The solution is to differentiate the effects that are in the frequency domain from those that are in the wavelength domain. In the analyzes conducted [12] over the gravitational and cosmological redshifts a $Z_{\text {CEF }}$ balance was suggested which weighs the different effects capable and liable on the trajectory of perform shifting the wavelength of light.

\subsection{RELATIVISTIC ENERGY WAVE (REW)}

The analysis conducted by Bhaduri et. al [6], which deals with space-time refraction (ST) reveal that in the transition between two media there will be a wave package that crosses the separating surface without changing the magnitude of the group velocity and another that retains the magnitude of the group velocity, but without group delay when traveling equal distances on both media, where the group speed can increase with the refractive index, as a function of the angle of incidence. ST wave packets represent part of the surface of a light cone, limited by the intersection of an isofrequency plane, or simply a spectral plan [6], [5], [30].

Recently, anomalies have been found in the refraction of ST wave packets [6], where the authors point out that traditional wave packets do not change the refraction group velocity, i.e., $\mathrm{v}_{\mathrm{g}}>$ c, implying that there will always be delays in the perception of distant receivers. In this perspective, the behavior of a photon in refraction according to the Snell-Descates Law and REW is verified in a comparative analysis to results of ST waves.

The REW presented in this study has characteristics similar to the ST wave, however the REW is associated with time dilation from the perspective of an observer in the source frame, whose relativistic effect is due to the relativistic ignition of the photon, associated with the Abraham momentum.

The deformations given by eq. (24) are independent of the thickness of the source and estimate the refracted angle as per Figure 2, where we verified its behavior by scanning incidence angles according to Bhaduri et al. [6], for comparison purposes although they are different degrees of freedom, where in Figure 4 regardless of wave packet geometry or group speed, REW explains that there are no speed anomalies in refringent media, but rather a relativistic effect that delays or advances the pointer from the perspective of the source referential, where the REW momentum is dilated and increases with the relative refraction index in accordance with Minkowski and the delay is a function of the angle of incidence.

In the representation of refraction by ST waves [6], the angular treatment is in relation to spectral plane tilt, however, when we are dealing here with a single photon, in the absence of a spectral plane, the inclination of the spectrum coincides with the angle of incidence of the photon, 
where we can conduct an analogous analysis weighing all the differences between the two descriptions.

Figure 4 presents the behavior of REW where the estimate of the angles of refraction is verified, according to eq. (24), in a spectrum of angles of incidence. It was not found for a single photon, anomalies presented by Bhaduri et al. [6] where $\theta_{2}>\theta_{1}$ in $n_{2}>n_{1}$, of which the authors associated the anomalies with group velocities and not with trajectories.

According to Kondaksi et al. [29], the ST waves feature acceleration of wave packets in air. The results presented here demonstrate that there are delays, synchronization, and advances between the clocks in the source and observer references. The REW wave characterizes the variability of time dilations, momentum and wavelength displacement, which can be seen in the results presented in the Figures 2 and 4, that the relativistic momentum of Minkowski shown here, by itself, is not able to compensate for delays as the compensation depends on the angle of incidence.

Checking the distance independency of source-observer synchronizations, as shown in Figure 4, it appears that the wave packets characterized by REW are able to connect source-receiver synchronously, precisely when the classical and relativistic trajectories overlap. The Snell-Descartes Law is not able to represent the behavior of data for large angles, as well as do not lay down a connection where delays are null between source-receiver, as predicted [6].

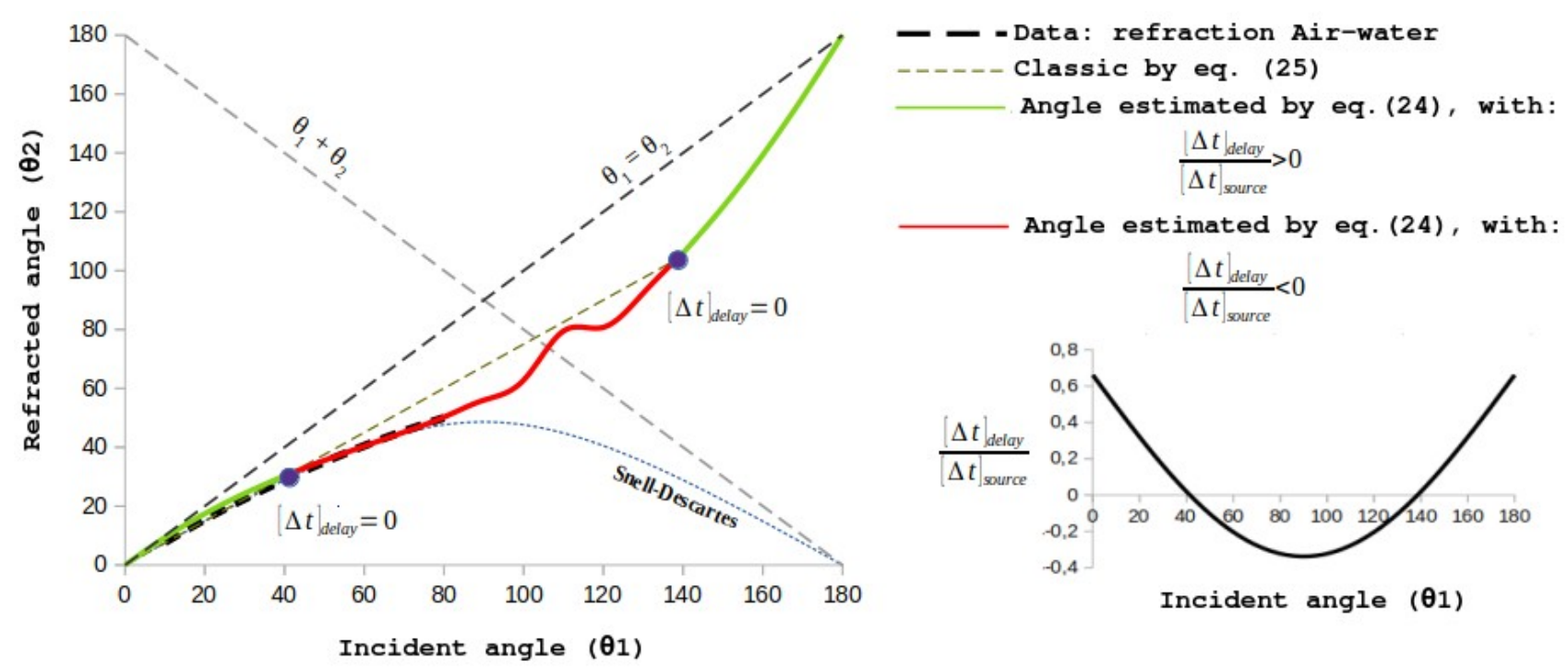

Figure 4- The graph on the left shows the REW delay and its refracted angle estimates and comparison with the experimental data according to Table 1 . The graph on the right shows the behavior of the REW delay as a function of the angle of incidence. (Source: author)

In the Figure 4, it is verified that the classical model given by eq. (25) which agrees with the Snell-Descates Law for small angles has a greater representation of the data behavior up to 
approximately $41^{\circ}$ of incidence, while the REW is delayed. The synchronization points demarcate the delay-advance inversion of the clock in the source referential, where the REW agrees with the classic model of eq. (25), but with the increase in the incidence angle, the photon starts to describe a relativistic trajectory with an advance of the clock in the reference of the source. For angles of incidence greater than $100^{\circ}$, the relativistic deformation is close to the classic deformation, where the clock in the reference of the source is slower and after synchronization the REW is delayed again, for large angles of incidence.

Although comparative analysis between ST and REW waves, we must consider that they deal with different refractions, in part. REW deals with refraction considering the actual trajectories of the wave packet, not finding any anomalies like those found in ST waves. The refraction anomalies found through ST waves are anomalies associated with group velocities within the scope of geometry adopted for the wave packet according to the authors [6], found in relation to the inclination of the spectral plane characteristic of the authors' approach.

In the Figure 5 the REW lag and lead zones are presented, as well as the refraction synchronizations for different pairs of media. For both pairs, in the incidence sloped in relation to normal, the delay is explained by the perception of time in the referential of the source and with the increase of the incidence slope, the source-observer synchronization/desynchronization occurs. In air the REW has no delay, except for slopes slightly close to normal.

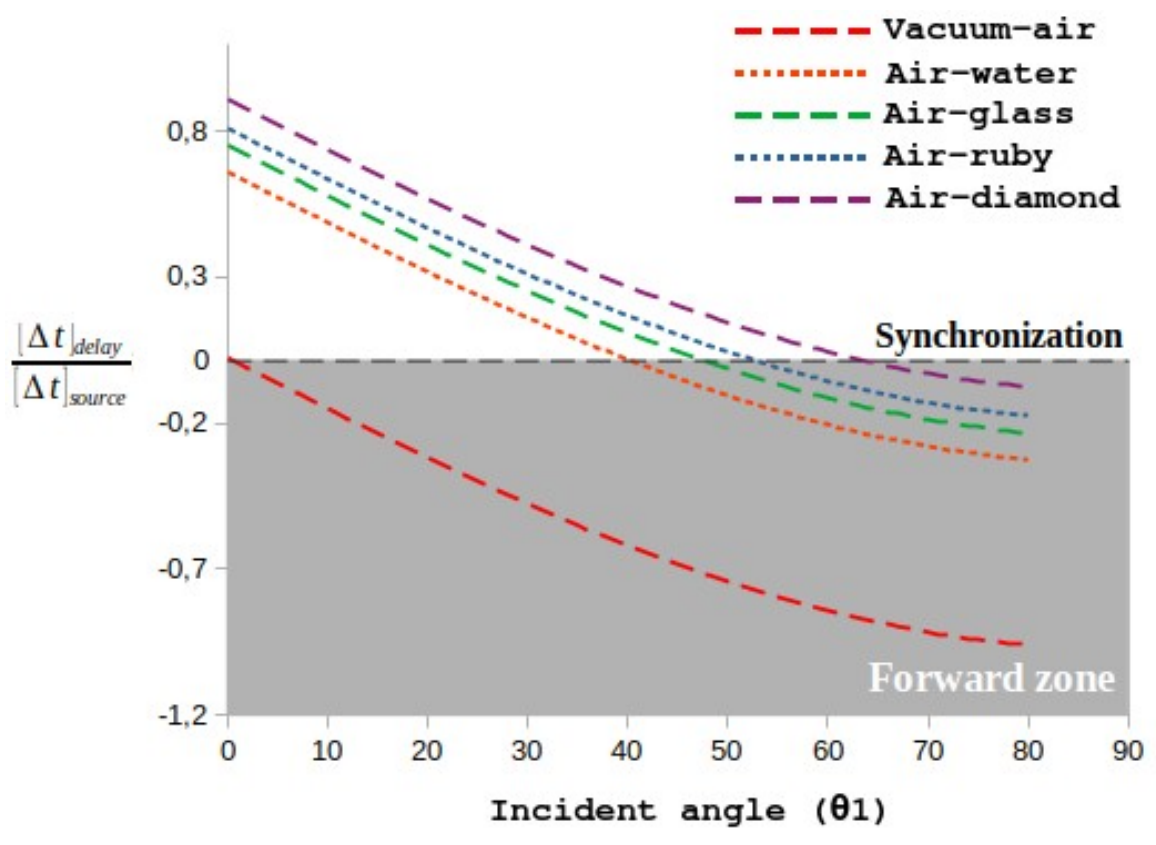

Figure 5-REW delays in the transition between pairs of materials (Source: author)

One might ponder that the relation of which we deal with the delay, eq. (36) is different from that treated in the studies discussed here [6], [33] and [46], as the authors divide the observation between before and after the separating surface, considering that the pulses will travel equal distances. In this study the delays presented by eq. (36) are independent of the source/observer 
distances in relation to the surface and consider the total sufficient time for the observer/source to perceive the source/observer.

\subsection{CONSERVATION OF ENERGY IN ANGULAR ORBITAL MOMENTUM (OAM)}

A photon or a very large number of photons passing through different media in a process where there is a shift in wavelength, keeping the frequency constant, will conserve energy in some form of movement. We show in eq.(s) (19) and (20) that the decrease of energy in the transition between two media is conserved in OAM, one characterized by Abraham's and the other by Minkowski, where we started to verify the variation of these momenta as a function of the angle of incidence.

Considering that the Abraham and Minkowski torques elapse during the delay interval, we can express the variation of the OAM from the perspective of the source's referential given by:

$$
\Delta L_{\text {source }}=\tau[\Delta t]_{\text {source }}=\frac{2 \hbar\left(1-n_{12}^{2}\right)}{\sqrt{1-\frac{v^{2}}{c^{2}}}-\operatorname{sen} \theta_{1}} .
$$

In the Figure 6, it can be seen from eq. (37) that the most pronounced variations of the OAM are found in the source-observer synchronizations, showing variation in sense of the OAM. It appears that OAM variations are very small, very close to zero, in the delayed/forward regions. in the air, in the Figure 6, it is possible to observe on a smaller scale that the most relevant variations of the OAM occur for small angles of incidence, close to normal.

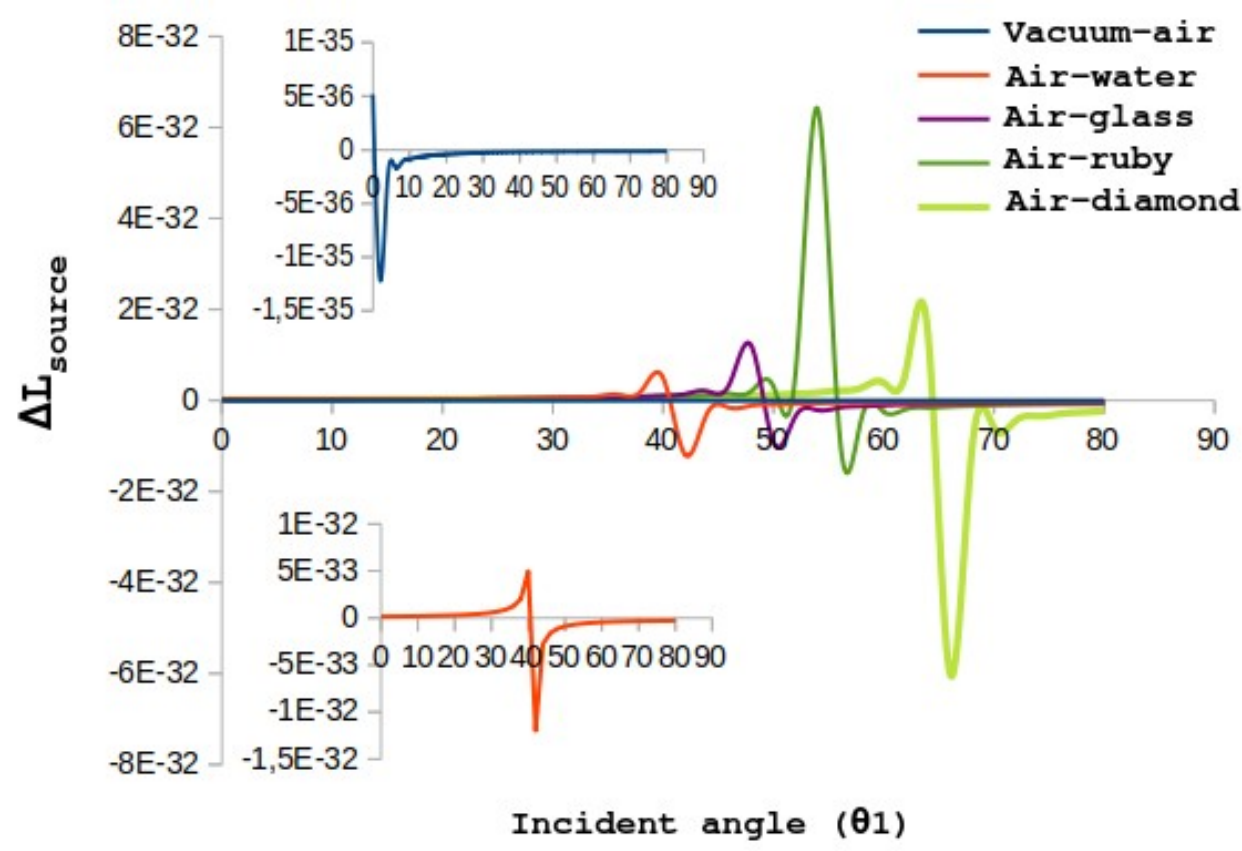

Figure 6-The OAM as a function of incidence angle, for the transition between pairs of media. (Source: author) 
The OAM variation signature shown in the Figure 6 is characteristic of its variability as treated by other authors [37], [25]. The intensities found in this study are small, whereas in this theoretical perspective the pulse is not previously modulated.

The variation of the OAM as a function of the angle of incidence characterized in the Figure 6 features new modulations. OAM modes are widely applied in quantum computing and information [11].

The conservation of photon mechanical energy in orbital angular movement discussed in this study, in agreement with Cardoso [13], [12], [10], describes a natural OAM of the photonmatter interaction, far from the perspective of artificial modulators. Recently, studies showed preliminary results of the OAM of photons emitted by natural sources [45] and theoretical and experimental results characterize the natural OAM(s) of ejection and photon-matter interaction, either by scattering [28].

\section{CONCLUSIONS}

It can be concluded in this study that the photon, when transiting between two media is subject to two non-additive torques, a classic associated with the Abraham momentum and a relativistic one characterized by the Minkowski momentum. The determination of the photon trajectory as classical or relativistic is a function of the incidence angle and the pair of refractive media, where the relativistic trajectory appears with delays.

The photon's energetic decrease, in the transition between two media, conditions the photon to Abraham's momentum, but also to a relativistic dynamic. Although it does not enjoy its common velocity in a vacuum, it moves at a velocity close to c, becoming a relativistic particle where its momentum and wavelength shift are dilated and the photon finds itself in a new state of movement, described by momentum of Minkowski, characterizing the momentum of Abraham as the hidden momentum of the relativistic momentum of Minkowski, while it appears as a relativistic photon ignition device.

An Apparent Doppler was found for a system where the source and observer are fixed, where the photon velocity in the refringent media characterizes the apparent relative velocity between source and observer, characterizing a Doppler effect for a constant frequency system, with wavelength shift.

The REW appears with a certain delay depending on the angle of incidence and the pair of media involved in the refraction scenario. In an analogue with ST waves, it was able to predict synchronization points between source and observer. The synchronization instants demarcate the alternations between REW delays and advances and also the agreement between classical and relativistic description. 
The conservation of the photon's mechanical energy in Orbital Angular Momentum indicates negligible variations while the REW presents delays and advances, inversion of the direction of the OAM in synchronization, indicating that the variations in the determination while classical or relativistic trajectories, is preceded by an inversion of the OAM. These OAM variations depend on the pair of media involved and the angle of incidence and may characterize new modulations for computation and quantum information.

\section{INDICATION OF POSSIBILITIES FOR FUTURE STUDIES}

Considering that the astigmatic behavior of light in refraction processes are acquaintances [4], [41], dealing with OAM-structured waves, where the pulse incident on a flat surface of a second refractive medium will present two contributions, one wave traveling the real path of the refracted beam and another local wave which has a path very close to the real path, although the slope relative to normal is slightly higher.

As well as, considering that in agreement with Gui et al. [26], an OAM - ST carrier pulse, second harmonic $(l=2)$ is distorted when being generated and propagated in crystals, associated with an ST astigmatism, consistent with the conservation of transverse OAM.

A study is suggested to verify the models dealt with in the present study, from the perspective of a pulse with AOM, where the astigmatic effect can be characterized by classical and relativistic trajectories determined by eq.(s) (24) and (25).

\section{REFERÊNCIAS}

[1] Allen, L., Beijersbergen, M. W., Spreeuw, R. J. C., \& Woerdman, J. P. (1992). Orbital angular momentum of light and the transformation of Laguerre-Gaussian laser modes. Physical Review A, 45(11), 8185-8189. doi:10.1103/physreva.45.8185.

[2] ASTRATH, Nelson GC et al. Unravelling the effects of radiation forces in water. Nature communications, v. 5, n. 1, p. 1-6, 2014.

[3] Barnett Stephen M. e Loudon Rodney, 2010. The enigma of optical momentum in a medium. Phil. Trans. R. Soc. A. 368 927-939.

[4] Baues, P. (1976). Gaussian beam parameter transformation at a refracting surface. Optics \& Laser Technology, 8(2), 85-88.doi:10.1016/0030-3992(76)90069-4.

[5] Bhaduri B., Murat Yessenov, and Ayman F. Abouraddy, "Space-time wave packets that travel in optical materials at the speed of light in vacuum," Optica 6, 139-146 (2019).

[6] Bhaduri, B., Yessenov, M. \& Abouraddy, AF Anomalous refraction of óptico spacetime wave packets. Nat. Photonics 14, 416-421 (2020). https://doi.org/10.1038/s41566$\underline{020-0645-6 .}$. 
[7] BOWYER, Peter. The momentum of light in media: the Abraham-Minkowski controversy. School of Physics \& Astronomy. Southampton, UK, 2005.

[8] C. Wang, "Can the Abraham Light Momentum and Energy in a Medium Constitute a Lorentz Four-Vector?,"Journal of Modern Physics, Vol. 4 No. 8, 2013, pp. 1123-1132. doi:10.4236/jmp.2013.48151.

[9] Cardona Suárez, C.A., Gutiérrez Valderrama, J. The law of refraction and Kepler's heuristics. Arch. Hist. Exact Sci. 74, 45-75 (2020). https://doi.org/10.1007/s00407-019-00236w

[10] CARDOSO, Daniel Souza. A conservação da energia mecânica do fóton, em energia cinética rotacional, frente à alguns resultados e expectativas teóricas com interferômetros de Michelson na literatura. Ciência e Natura, v. 40, p. e59, 2018.

[11] CARDOSO, Daniel Souza. A NATUREZA DO MOMENTO ANGULAR ORBITAL DO FÓTON COMO PROPRIEDADE DA INTERAÇÃO FÓTON-MATÉRIA. Revista Sociedade Científica, v. 2, n. 4, p. 1-12, 2019.

[12] CARDOSO, Daniel Souza. A NATUREZA DO REDSHIFT SEGUNDO O PRINCÍPIO DE CONSERVAÇÃO DA ENERGIA MECÂNICA DO FÓTON. Revista Sociedade Científica 4 (1), 1-21

[13] CARDOSO, Daniel Souza. Theory of Conservation of Photon Mechanical Energy , in the Transition between Two Middles , in Rotational Kinetic Energy. International Journal of Science and Research (IJSR) 7 (7), 810-815, 2018.

[14] CARROLL, Bradley W.; AMIRI, Farhang; GALLI, J. Ronald. An Effective Photon Momentum in a Dielectric Medium: A Relativistic Approach. arXiv preprint arXiv:1801.00679, 2017.

[15] CATALDO, Carmine et al. A short discussion on the Gravitational Redshift in the light of an alleged local variability of the Planck Constant. Journal of Applied Mathematics and Physics, v. 5, n. 05, p. 1001, 2017.

[16] Chen, J., Dai, Y. \& Xuanyuan, Y. Possible solution of Abraham-Minkowski controversy by generalizing the principle of invariance of light speed. $J$ Opt 49, 127-131 (2020). https://doi.org/10.1007/s12596-020-00586-7.

[17] Curtis, J. E., \& Grier, D. G. (2003). Structure of Optical Vortices. Physical Review Letters, 90(13). doi:10.1103/physrevlett.90.133901.

[18] DE Broglie, Louis. The wave nature of the electron. Nobel lecture, v. 12, p. 244-256, 1929.

[19] DE OLIVEIRA FILHO, Juvenil Siqueira. Momento oculto em sistemas quânticos e sua relação com o momento da luz na matéria. 2016. 
[20] EINSBERG, Robert; RESNICK, Robert. Física Quântica. Rio de Janeiro: Elsevier, ISBN 85-700-1309-4, 24 reimpressão, p. 319, 1979.

\section{[21] F. Zwicky. ON THE REDSHIFT OF SPECTRAL LINES THROUGH}

INTERSTELLAR SPACE .Proceedings of the National Academy of Sciences Oct 1929, 15 (10) 773-779; DOI: 10.1073/pnas.15.10.773.

[22] FORBES, Kayn A.; ANDREWS, David L. Orbital angular momentum of twisted light: chirality and optical activity. Journal of Physics: Photonics, 2021.

[23] Giovannini, D., Romero, J., Poto ek, V., Ferenczi, G., Speirits, F., Barnett, S. M., ... Padgett, M. J. (2015). Spatially structured photons that travel in free space slower than the speed of light. Science, 347(6224), 857-860.

[24] GORAY, Mahendra; ANNAVARAPU, Ramesh Naidu. Rest mass of photon on the surface of matter. Results in Physics, v. 16, p. 102866, 2020.

[25] Götte, J. B., \& Dennis, M. R. (2013). Limits to superweak amplification of beam shifts. Optics Letters, 38(13), 2295. doi:10.1364/ol.38.002295.

[26] Gui, G., Brooks, N.J., Kapteyn, H.C. et al. Second-harmonic generation and the conservation of spatiotemporal orbital angular momentum of light. Nat. Photon. 15, 608613 (2021). https://doi.org/10.1038/s41566-021-00841-8.

[27] JI, Zhurun et al. Photocurrent detection of the orbital angular momentum of light. Science, v. 368, n. 6492, p. 763-767, 2020.

[28] Katoh, M., Fujimoto, M., Mirian, N.S. et al. Helical Phase Structure of Radiation from an Electron in Circular Motion. Sci Rep 7, 6130 (2017). https://doi.org/10.1038/s41598017-06442-2.

[29] Kondakci, H. E., \& Abouraddy, A. F. (2018). Airy Wave Packets Accelerating in Space-Time. Physical Review Letters, 120(16).doi:10.1103/physrevlett.120.163901.

[30] Kondakci, HE, Abouraddy, AF Optical space-time wave packets having arbitrary group velocities in free space. Nat Commun 10, 929 (2019). https://doi.org/10.1038/s41467019-08735-8.

[31] MANSURIPUR, Masud. Radiation pressure and the linear momentum of light in dispersive dielectric media. Optics Express, v. 13, n. 6, p. 2245-2250, 2005.

[32] Martin P. J. Lavery, Stephen M. Barnett, Fiona C. Speirits, and Miles J. Padgett, "Observation of the rotational Doppler shift of a white-light, orbital-angular-momentumcarrying beam backscattered from a rotating body," Optica 1, 1-4 (2014).

[33] MOTZ, Alyssa M. Allende et al. Refraction of space-time wave packets: II.

Experiments at normal incidence. arXiv preprint arXiv:2104.12969, 2021. 
[34] NEVES, Marcos Cesar Danhoni. A QUESTÃo CONTROVERSA DA COSMOLOGIA MODERNA: HUBBLE E O INFINITO parte 1. CADERNO CATARINENSE DE ENSINO DE FÍSICA, p. 189, 2000.

[35] Nikolai B. Chichkov and Boris N. Chichkov, "On the origin of photon mass, momentum, and energy in a dielectric medium [Invited]," Opt. Mater. Express 11, 27222729 (2021)

[36] NUSSEnZVEIG, H. Moysés. Física Básica 4, São Paulo: Ed. Blücher, ISBN 85-2120163-X, vol. 4, 4 ed., 437 p., 2002

[37] Ou, J., Jiang, Y., Zhang, J., \& He, Y. (2013). Reflection of Laguerre-Gaussian beams carrying orbital angular momentum: a full Taylor expanded solution. Journal of the Optical Society of America A, 30(12), 2561.doi:10.1364/josaa.30.002561.

[38] Pablo L. Saldanha, "Division of the momentum of electromagnetic waves in linear media into electromagnetic and material parts," Opt. Express18, 2258-2268 (2010) [39] PADGETT, Miles; BARNETT, Stephen M.; LOUDON, Rodney. The angular momentum of light inside a dielectric. journal of modern optics, v. 50, n. 10, p. 1555-1562, 2003.

[40] Partanen, M., Häyrynen, T., Oksanen, J., \& Tulkki, J. (2017). Photon mass drag and the momentum of light in a medium. Physical Review A, 95(6). doi:10.1103/physreva.95.063850.

[41] RITBOON, Atirach. The effect of astigmatism induced by refraction on the orbital angular momentum of light. Journal of Optics, v. 22, n. 7, p. 075201, 2020.

[42] Schmiegelow, C., Schulz, J., Kaufmann, H. et al. Transfer of optical orbital angular momentum to a bound electron. Nat Commun 7, 12998 (2016).

https://doi.org/10.1038/ncomms12998.

[43] Shapiro, I. I. (1964). Fourth Test of General Relativity. Physical Review Letters, 13(26), 789-791. doi:10.1103/physrevlett.13.789

[44] Tan, C. Z. (2015). Imaginary rest mass of a photon in a dispersive medium. Optik International Journal for Light and Electron Optics, 126(24), 5304-5306. doi:10.1016/j.ijleo.2015.09.009

[45] URIBE-PATARROYO, N. et al. Detecting photons with orbital angular momentum in extended astronomical objects: application to solar observations. Astronomy \& Astrophysics, v. 526, p. A56, 2011.

[46] YESSENOV, Murat et al. Refraction of space-time wave packets: III. Experiments at oblique incidence. arXiv preprint arXiv:2104.12972, 2021. 
[47] Zhang, C., Xu, P., \& Jiang, X. (2020). Vortex electron generated by microwave photon with orbital angular momentum in a magnetic field. AIP Advances, 10(10), 105230. doi:10.1063/5.0019899. 